\title{
Fasudil regulates $T$ cell responses through polarization of BV-2 cells in mice experimental autoimmune encephalomyelitis
}

\author{
Chan CHEN ${ }^{1}$, Yan-hua $\mathrm{LI}^{2}$, Qiong ZHANG ${ }^{1}$, Jie-zhong $\mathrm{YU}^{2}$, Yong-fei ZHAO ${ }^{1}$, Cun-gen $\mathrm{MA}^{2,3}$ * , Bao-guo XIAO ${ }^{1, *}$ \\ ${ }^{1}$ Institute of Neurology, Huashan Hospital, Institutes of Brain Science and State Key Laboratory of Medical Neurobiology, Fudan \\ University, Shanghai 200040, China; ${ }^{2}$ Institute of Brain Science, Department of Neurology, Medical School, Shanxi Datong University, \\ Datong 037009, China; ${ }^{3}$ Department of Encephalopathy and National Major Clinical Department of Ministry of Health, Third Hospital, \\ Shanxi University of Traditional Chinese Medicine, Taiyuan 030006, China
}

Aim: Fasudil, a selective Rho kinase (ROCK) inhibitor, has been shown to alleviate the severity of experimental autoimmune encephalomyelitis (EAE) via attenuating demyelination and neuroinflammation. The aim of this study was to investigate the effects of fasudil on interactions between macrophages/microglia and T cells in a mice EAE model.

Methods: Mouse BV-2 microglia were treated with IFN-y and fasudil. Cell viability was detected with MTT assay. BV-2 microglia polarization was analyzed using flow cytometry. Cytokines and other proteins were detected with ELISA and Western blotting, respectively. Mice were immunized with $\mathrm{MOG}_{35-55}$ to induce $\mathrm{EAE}$, and then treated with fasudil (40 mg/kg, ip) every other day from d 3 to d 27 pi. Encephalomyelitic T cells were prepared from the spleen of mice immunized with $\mathrm{MOG}_{35-55}$ on d 9 pi.

Results: Treatment of mouse BV-2 microglia with fasudil $(15 \mu \mathrm{g} / \mathrm{mL})$ induced significant phenotype polarization and functional plasticity, shifting M1 to M2 polarization. When co-cultured with the encephalomyelitic T cells, fasudil-treated BV-2 microglia significantly inhibited the proliferation of antigen-reactive T cells, and down-regulated IL-17-expressing CD4+ $\mathrm{T}$ cells and IL-17 production. Furthermore, fasudil-treated BV-2 microglia significantly up-regulated CD4 ${ }^{+} \mathrm{CD} 25^{\text {high }}$ and CD4 ${ }^{+} \mathrm{IL}-10^{+}$regulatory T cells (Tregs) and IL-10 production, suggesting that the encephalomyelitic $T$ cells had converted to Tregs. In EAE mice, fasudil administration significantly decreased both $\mathrm{CD}_{11} \mathrm{~b}^{+} \mathrm{iNOS}^{+}$and CD11 $\mathrm{b}^{+} \mathrm{TNF}-\alpha^{+} \mathrm{M} 1$ microglia, and increased CD11 $\mathrm{b}^{+} \mathrm{IL}-10^{+} \mathrm{M} 2$ microglia.

Conclusion: Fasudil polarizes BV-2 microglia into M2 cells, which convert the encephalomyelitic T cells into Tregs in the mice EAE model.

Keywords: fasudil; multiple sclerosis; experimental autoimmune encephalomyelitis; neuroinflammation; microglia; T cell; cell polarization; cytokine

Acta Pharmacologica Sinica (2014) 35: 1428-1438; doi: 10.1038/aps.2014.68; published online 29 Sep 2014

\section{Introduction}

Multiple sclerosis (MS) is the most common multifocal inflammatory demyelination occurring in the white matter of the central nervous system (CNS). It is characterized by myelin sheath loss and consequent axon and neuron injury ${ }^{[1]}$. Experimental autoimmune encephalomyelitis (EAE) shares many pathological and histological similarities with MS and is, therefore, widely used as a preclinical model of human disease $e^{[2,3]}$. Th1 cells have been considered as primary effector $\mathrm{T}$ cells that participate in the pathogenetic process of $\mathrm{MS}^{[4]}$. Recent stud-

\footnotetext{
* To whom correspondence should be addressed.

E-mail bgxiao@shmu.edu.cn (Bao-guo XIAO); macungen2001@163.com (Cun-gen MA)

Received 2014-01-13 Accepted 2014-04-22
}

ies have indicated that Th17 cells are another major subtype of pathogenic $\mathrm{T}$ cells involved in the development of $\mathrm{EAE}^{[5]}$. Additionally, within the CNS, the activation and function of astrocytes and microglia are critical in the pathogenesis of $\mathrm{EAE}^{[6,7]}$. In chronic inflammatory pathogenesis, such as MS/ EAE, a complex interplay between Th1, Th17, Th2 and regulatory $\mathrm{T}$ cells (Tregs) is believed to determine the outcome of the disease $^{[4]}$. Therefore, the respective conversion of these cell populations and the discovery of novel agents that promote the expansion of beneficial cell populations may be critical to develop new therapeutic strategies against chronic inflammatory diseases such as MS.

In the CNS, microglia play essential roles in the maintenance of homeostasis and responses to neuroinflammation ${ }^{[8,9]}$ and are considered important contributors to tissue damage 
in the pathogenesis of MS/EAE for several reasons, including the following: 1) there is a strong correlation between microglia activity and both axonal injury ${ }^{[10]}$ and oligodendrocyte $\operatorname{loss}^{[11]}$; 2) microglia-conditioned medium can kill oligodendrocytes in culture ${ }^{[12]}$; and 3) the inhibition of macrophages/ microglia activity with clodronate liposomes or genetic deletion alleviates disease severity in $\mathrm{EAE}^{[10,13]}$. However, in addition to these detrimental effects, microglia have beneficial roles within the $\mathrm{CNS}^{[6,14,15]}$. In recent years, microglia, similar to macrophages, have been subdivided into M1 and M2 phenotypes ${ }^{[16,17]}$. M1 microglia/macrophages are considered proinflammatory, producing proinflammatory cytokines such as TNF-a. M2 microglia/macrophages are regulatory/ anti-inflammatory and secrete regulatory cytokines such as interleukin (IL)-10. The M2 subset could prominently dampen EAE severity, demonstrating a beneficial effect of the M2 phenotype ${ }^{[18]}$.

During the course of EAE, demyelination and axonal loss are mediated by the interplay between microglia and reactive $\mathrm{T}$ cells $\mathrm{s}^{[19]}$. Upon encountering the myelin antigen, activated microglia present the antigen to $\mathrm{T}$ cells and trigger the re-activation of infiltrated $\mathrm{T}$ cells, leading to disease progression $^{[8,20]}$. As microglia are involved in multiple stages of MS/EAE pathology, it makes microglia a potential therapeutic target to study and manipulate.

Fasudil [1-(5-isoquinolinesulfonyl)-homo-piperazine], a selective Rho kinase (ROCK) inhibitor, has been used to treat cerebral vasospasm ${ }^{[21]}$, stroke ${ }^{[22]}$, and traumatic spinal cord injury ${ }^{[23]}$. Our previous study showed that fasudil alleviated the severity of EAE and attenuated demyelination and neuroinflammation, acting possibly through an anti-inflammatory mechanism ${ }^{[24]}$. In addition, oral administration of fasudil prevented the development of EAE induced by proteolipid protein (PLP) p139-151 in SJL/J mice ${ }^{[25]}$. The mechanism of action might be the reduction of lymphocyte proliferation triggered by specific PLPs and the down-regulation of IL-17 levels and the IFN- $\gamma /$ IL-4 ratio $^{[25]}$. Moreover, we observed that the polarization of the M1 macrophage phenotype to the M2 phenotype in fasudil-treated EAE mice was accompanied by the inhibition of Th17 $\mathrm{T}$ cells and an increase in Th2 $\mathrm{T}$ cells ${ }^{[26]}$. Although several publications describe the therapeutic effects and immunological changes after fasudil treatment in EAE models, little is known regarding the immunomodulatory interaction of fasudil with microglia and T cells. In the present study, we tested the hypothesis that fasudil modifies the polarization of microglia, which further convert encephalomyelitic $\mathrm{T}$ cells into Tregs.

\section{Materials and methods}

\section{Animals and ethics statement}

Female C57BL/ 6 mice (8-10 weeks old and 18-20 g) were purchased from Vital River Laboratory Animal Technology Co Ltd, Beijing, China. The study was approved by the Ethics Committee of Shanxi Datong University, Datong, China, and it was performed in accordance with the ethical standards of the International Council for Laboratory Animal Science. All mice were housed under pathogen-free conditions and maintained in a reversed 12:12-h light/dark cycle in a temperaturecontrolled room $\left(25 \pm 2^{\circ} \mathrm{C}\right)$ for 1 week prior to experimental manipulation.

\section{BV-2 microglial culture}

The BV-2 immortalized microglial cell line ${ }^{[27]}$ was purchased from ShenKe Biological Technology Co, Ltd, Shanghai, China, and cultured in Dulbecco's modified Eagle's medium (DMEM; Gibco/Life Technologies, Carlsbad, CA, USA), supplemented with 10\% fetal bovine serum (FBS; Gibco/Life Technologies, Carlsbad, CA, USA), $100 \mathrm{U} / \mathrm{mL}$ penicillin and $100 \mu \mathrm{g} / \mathrm{mL}$ streptomycin (Gibco/Life Technologies, Carlsbad, CA, USA) at $37^{\circ} \mathrm{C}$ in a humidified cell incubator. The cells were plated on 96-well, 24-well, or 6-well plates at a density of $1 \times 10^{6} / \mathrm{mL}$ for the experiments. For the subculture, the cells were removed from the culture flask, re-suspended in complete medium and sub-cultured in different plates for the experiments.

\section{Induction of chronic EAE}

Mouse myelin oligodendrocyte glycoprotein peptide ${ }_{35-55}$ (MOG $35-55$, MEVGWYRSPFSRVVHLYRNGK) was produced in an automatic synthesizer (CL Bio-Scientific Company, Xi-an, China). The purity of the peptide was $>95 \%$ as determined by HPLC.

EAE was induced by immunization with $300 \mu \mathrm{g}$ of $\mathrm{MOG}_{35-55}$ in Freund's complete adjuvant (Sigma, St Louis, MO, USA) supplemented with $3 \mathrm{mg} / \mathrm{mL}$ of tuberculosis H37Ra (BD Difco, Detroit, MI, USA) $(400 \mu \mathrm{g} /$ mice $)$. Mice were intraperitoneally (ip) injected with $750 \mathrm{ng}$ of pertussis toxin (Enzo Life Sciences, Farmingdale, NY, USA) on d 0 and 2 postimmunization (pi). Animals were weighed and evaluated for clinical score in a blinded fashion by at least two investigators. Clinical score of EAE was graded according to the following criteria: 0 , healthy; 1 , limp tail; 2 , ataxia and/or paresis of hind limbs; 3, paralysis of hind limbs and/or paresis of forelimbs; 4, tetraparalysis; and 5, moribund or death.

\section{Administration of fasudil}

Mice were divided into 2 groups, the fasudil experimental group and the saline control group ( $n=8$ in each group). Fasudil (Tianjin Chase Sun Pharmaceutical Co, Ltd, Tianjin, China) was injected ip at $40 \mathrm{mg} \cdot \mathrm{kg}^{-1} \cdot \mathrm{d}^{-1}$ every other day from $\mathrm{d}$ 3 to $\mathrm{d} 27 \mathrm{pi}$. The injection of saline was set up as a control in a similar manner.

\section{Cell viability assay}

Cell viability was detected by MTT assay. Briefly, $100 \mu \mathrm{L}$ of 3-(4,5-dimethylthiazol-2-yl)-2,5-diphenyltetrazolium bromide (MTT) solution $(0.5 \mathrm{mg} / \mathrm{mL})$ was added to the cultured cells and incubated for $4 \mathrm{~h}$ at $37^{\circ} \mathrm{C}$. After adding $100 \mu \mathrm{L}$ DMSO, the $O D$ value $(570 \mathrm{~nm})$ was detected by a Synergy H1 Hybrid reader (BioTek Instruments, Winooski, VT, USA).

\section{LDH assay}

Cell death was measured by lactate dehydrogenase (LDH) 
release. The levels of $\mathrm{LDH}$ in the supernatants of the cultured cells were measured with a cytotoxicity detection kit (Promega, Madison, WI, USA), according to the manufacturer's protocol. The maximum LDH release assessed by freeze-thaw lysing the normal culture cells was considered as the control value (100\% LDH release). The data were expressed as the percentage of the control value.

\section{Preparation of encephalomyelitic T cells}

The encephalitogenic CD4-positive T cells specific for $\mathrm{MOG}_{35-55}$ were obtained from the spleen of mice that had been immunized with $\mathrm{MOG}_{35-55}$. The mice were immunized with $\mathrm{MOG}_{35-55}$ using the procedure used for EAE induction. On $\mathrm{d}$ $9 \mathrm{pi}$, the spleen was removed and the mononuclear cells were prepared. The encephalitogenic CD4-positive T cells specific for $\mathrm{MOG}_{35-55}$ were obtained with the use of anti-mouse CD4 magnetic beads (Miltenyi Biotech, Auburn, CA, USA).

\section{Co-culture of BV-2 and encephalomyelitic T cells}

The BV-2 cells were cultured in the absence or presence of IFN-Y $(100 \mathrm{U} / \mathrm{mL})$ and/or fasudil $(15 \mu \mathrm{g} / \mathrm{mL})$ for $72 \mathrm{~h}$. Next, IFN- $\gamma$ and fasudil were removed by washing with PBS before co-culturing the BV-2 cells with the encephalomyelitic T cells. The encephalomyelitic T cells were added to the BV-2 monolayer (fasudil- or PBS-treated BV-2 cells) at an estimated ratio of 5:1 in complete medium supplemented with $\mathrm{MOG}_{35-55}$ $(10 \mu \mathrm{g} / \mathrm{mL})$ and were maintained in co-culture for $72 \mathrm{~h}$. The supernatants were collected to analyze nitric oxide and cytokines, and the cells were obtained for flow cytometric analysis.

\section{Flow cytometric analysis}

The BV-2 cells were stained with the following antibodies: PE-CD16/32, PE-CD206, PE-CCR7, PE-CD11c, PE-CD23, PEMHC class II (eBioscience, San Diego, CA, USA), anti-iNOS and anti-arginase (BD Biosciences, Franklin Lakes, NJ, USA), followed by the corresponding PE-conjugated secondary antibodies for iNOS and arginase. The mixed cells from the co-culture were stained with the following antibody pairs: FITC-CD4 and PE-CD25 (eBioscience, San Diego, CA, USA), PE-IFN- $\gamma$, PE-IL-10, and PE-IL-17 (eBioscience, San Diego, CA, USA). The cells were gated using forward and sideward scatter characteristics for lymphocytes and monocytes, and at least 10000 gated events were collected using flow cytometer (BD Biosciences, Franklin Lakes, NJ, USA). The data were analyzed using CellQuest software.

\section{T cell proliferation assay}

The T cells were labeled with carboxyfluorescein diacetate succinimidyl ester (CFSE) and co-cultured with PBS- or fasudiltreated BV-2 microglia (T cells: BV-2 microglia, 5:1) in the presence of $\mathrm{MOG}_{35-55}(10 \mu \mathrm{g} / \mathrm{mL})$. After $72 \mathrm{~h}$, the number of proliferating cells was determined through CFSE dilution with flow cytometry. The data were analyzed using CellQuest software.

\section{Cytokine ELISA assay}

The supernatants of the T cell and BV-2 microglia co-culture were harvested and analyzed for the cytokine concentrations of IFN-y, IL-10, IL-17, and TNF-a using sandwich ELISA kits (Peprotech, Rocky Hill, NJ, USA), following the manufacturer's instructions. The concentrations of the cytokines were quantified with reference to the standard curve. The measurements were performed in duplicate and repeated as 3 independent experiments. The results were expressed as $\mathrm{pg} / \mathrm{mL}$.

\section{Immunohistochemistry}

The mice were anesthetized and perfused with PBS and $4 \%$ buffered paraformaldehyde. Sections of the spinal cord $(10 \mu \mathrm{m})$ were incubated with anti-CD11b (1:1000, eBioscience, San Diego, CA, USA) at $4{ }^{\circ} \mathrm{C}$ overnight and with Alexa Fluor 488-conjugated secondary antibodies (1:1000, Invitrogen, Eugene, OR, USA) at room temperature (RT) for $2 \mathrm{~h}$. The slides were incubated at $4{ }^{\circ} \mathrm{C}$ overnight with anti-iNOS (1:1000, BD Biosciences, Franklin Lakes, NJ, USA), anti-TNF- $\alpha$ (1:500, Peprotech, Rocky Hill, NJ, USA), anti-arginase (1:1000, BD Biosciences, Franklin Lakes, NJ, USA) or anti-IL-10 (1:500, Peprotech, Rocky Hill, NJ, USA), followed by Alexa Fluor 555-conjugated secondary antibodies (1:1000, Invitrogen, Eugene, OR, USA) at RT for $2 \mathrm{~h}$. As a negative control, additional sections were treated similarly, without the primary antibodies.

\section{Western blot analysis}

The BV-2 microglia were homogenized with a glass homogenizer using ice-cold lysis buffer supplemented with protease inhibitors. The protein content was quantitated using the Bradford protein assay (Sigma, St Louis, MO, USA). The lysates were loaded on SDS-polyacrylamide gels and transferred onto nitrocellulose membrane (Merck Millipore, Tullagreen Carrigtwohill, Cork, Ireland). The membranes were then incubated at $4{ }^{\circ} \mathrm{C}$ overnight with antibodies against antiiNOS (1:1000, BD Biosciences, Franklin Lakes, NJ, USA), antiarginase (1:1000, BD Biosciences, Franklin Lakes, NJ, USA) and anti-GAPDH (1:1000, Epitomics, Burlingame, CA, USA). Next, the membranes were incubated with the horseradish peroxidase-conjugated secondary antibodies (Cell Signaling Technology, Danvers, MA, USA) for $1 \mathrm{~h}$ at RT. The immunoblots were developed using an enhanced chemiluminescence (ECL) system (GE Healthcare, Buckinghamshire, UK) and analyzed using Quantity Software (Bio-Rad, Hercules, CA, USA).

\section{Nitrite assay}

Nitric oxide (NO) was assayed by measuring the end product nitrite, which was determined by a colorimeter assay based on the Griess reaction. The supernatants $(100 \mu \mathrm{L})$ of the cultured cells were mixed with $100 \mu \mathrm{L}$ of Griess reagent for $10 \mathrm{~min}$ at RT. The absorbance was measured at $510 \mathrm{~nm}$. The concentrations of nitrite were determined with reference to a standard curve of sodium nitrite (Sigma, St Louis, MO, USA). The results were expressed as $\mu \mathrm{mol} / \mathrm{L}$. 
Arginase assay

The arginase activity was measured using the QuantiChrom ${ }^{\mathrm{TM}}$ arginase assay kit (BioAssay Systems, Hayward, CA, USA) following the manufacturer's instructions. Briefly, $40 \mu \mathrm{L}$ sample was mixed with or without (blank control) $10 \mu \mathrm{L}$ substrate buffer at $37^{\circ} \mathrm{C}$ for $2 \mathrm{~h}$. In addition, $50 \mu \mathrm{L}$ of $1 \mathrm{mmol} / \mathrm{L}$ urea standard was used to prepare the standard curve. Subsequently, $200 \mu \mathrm{L}$ urea reagent and $10 \mu \mathrm{L}$ substrate were added to all wells and incubated for $60 \mathrm{~min}$ at RT. The optical density was measured at $430 \mathrm{~nm}$. The arginase activity was calculated as: $\left(O D_{\text {sample }}-O D_{\text {blank }} / O D_{\text {standard }}-O D_{\text {water }}\right) \times 10.4$, and expressed as $\mathrm{U} / \mathrm{L}$.

\section{Statistical analysis}

Multiple comparisons among the experimental groups were performed using ANOVA followed by post hoc tests. Comparisons between two experimental groups were made using Student's $t$ test. $P<0.05$ was considered statistically significant.

\section{Results}

The effect of fasudil on the morphology and cell viability of BV-2 microglia

Resting microglia were characterized by rounded and elongated cell bodies with irregular shape (some tripolar), whereas fasudil treatment stimulated long dendritic formations in the BV-2 microglial morphology (Figure 1A). We measured cell viability $24 \mathrm{~h}$ after fasudil treatment using MTT and LDH assays. No change was observed in the MTT (Figure 1B) and LDH assay results (Figure 1C) after exposure to 1, 5, and 15 $\mu \mathrm{g} / \mathrm{mL}$ of fasudil. However, there was a marked decrease in cell viability and increase in cell death after exposure to 50 $\mu \mathrm{g} / \mathrm{mL}$ of fasudil, indicating that a higher dose of fasudil is cytotoxic.

We further examined the morphological changes and cell viability $24 \mathrm{~h}$ after treating the BV-2 cells with IFN-ץ (100 $\mathrm{U} / \mathrm{mL})$ and/or fasudil $(15 \mu \mathrm{g} / \mathrm{mL})$. As shown in Figure 1D, no obvious morphological change was observed in the BV-2 microglia treated with PBS and IFN- $\gamma$, and the fasudil treatment stimulated long dendritic formations. However, there were no differences in the cell viability after exposure to 15 $\mu \mathrm{g} / \mathrm{mL}$ of fasudil (Figure $1 \mathrm{E}$ and $1 \mathrm{~F}$ ).

\section{Fasudil shifts M1 to M2 phenotype of BV-2 microglia in vitro}

To characterize the effect of fasudil on the functions of the BV-2 microglia, we first measured the polarization of the BV-2 cells with the M1 markers iNOS, MHC class II, CD11c, CCR7, and CD16/32, as well as the M2 markers arginase, CD23 and CD206 by flow cytometry. Phenotypic analysis of the BV-2 cells showed that the expression of the M1 markers iNOS, MHC class II, CD11c, and CD16/32 declined after treating the BV-2 cells with IFN-Y $(100 \mathrm{U} / \mathrm{mL})$ and fasudil (15 $\mu \mathrm{g} / \mathrm{mL}$ ), compared with that after the IFN- $\gamma$ treatment (Figure 2, $P<0.01$, respectively). The expression of CCR7 was not significantly different among the three groups. By contrast, 3 M2 markers arginase, CD23 and CD206 were elevated after the IFN-ץ plus fasudil treatment; however, only the expression
A
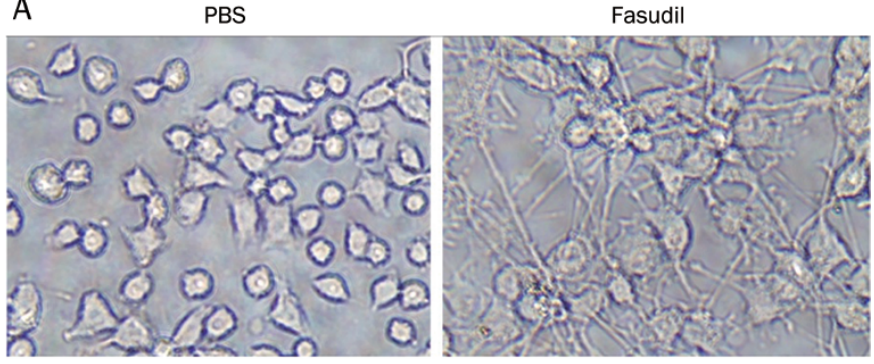

B

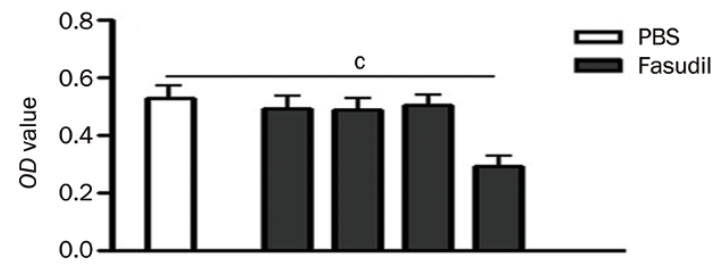

C

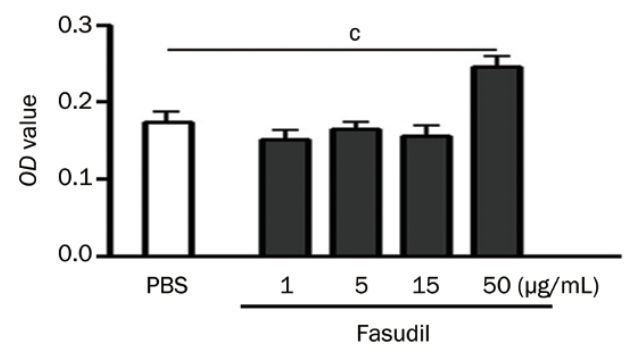

D
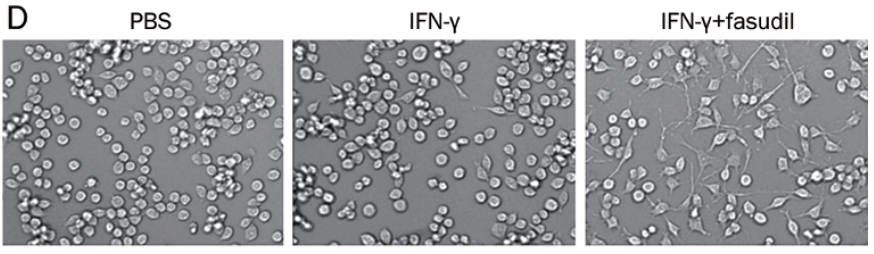

$\mathrm{E}$
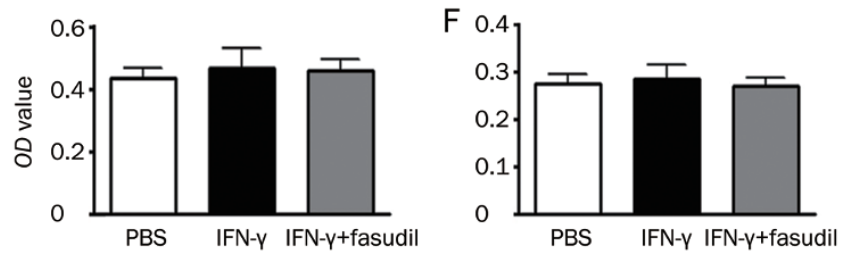

Figure 1. Morphology and viability of BV-2 microglia treated with fasudil in vitro. The BV-2 cells were cultured in 24-well and 96-well plates in the absence or presence of IFN-y $(100 \mathrm{U} / \mathrm{mL})$ and/or fasudil $(1,5,15$, and $50 \mu \mathrm{g} / \mathrm{mL}$ ) for $24 \mathrm{~h}$. The cell morphology was obtained by bright field imaging using an inverted Olympus microscope. The photomicrographs are representative pictures depicting the BV-2 microglia (A and D). Cell viability was measured using the MTT assay (B and E), and cell death was detected by $\mathrm{LDH}$ release assay ( $\mathrm{C}$ and $\mathrm{F}$ ). The quantitative analysis was performed based on 3 independent experiments with similar results. ${ }^{\mathrm{c}} P<0.01$.

of CD206 was statistically significant (Figure 2, $P<0.05$ ). The treatment of fasudil alone seemed to reduce the expression of M1 markers, such as iNOS, MHC II, and CD11c, compared with the treatment with PBS; however, only the decrease in CD11c expression was statistically significant (Figure 2, 

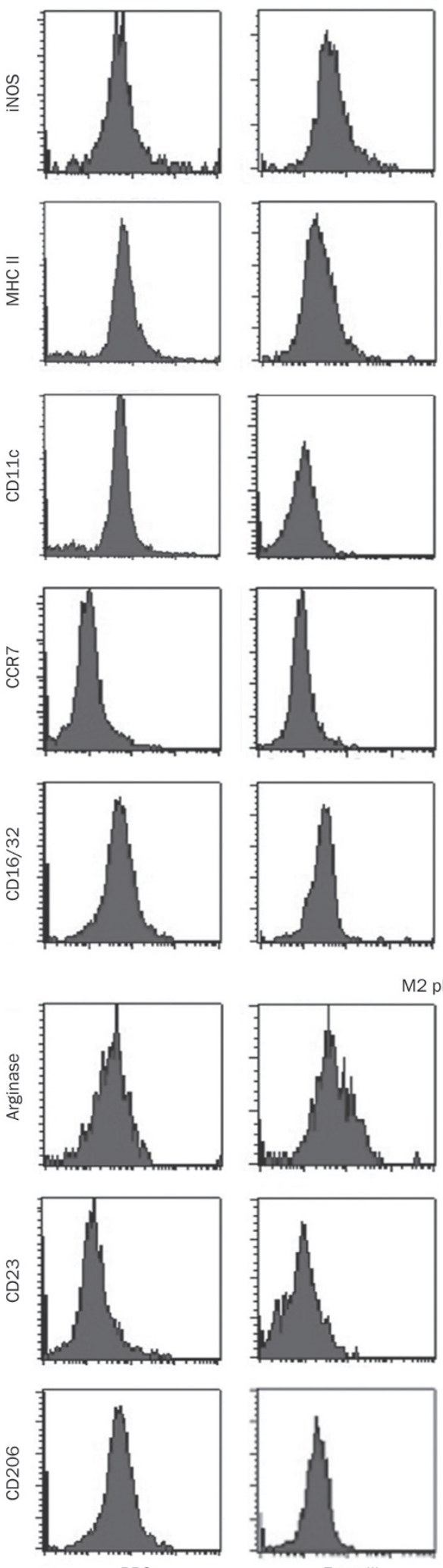

Fasudil
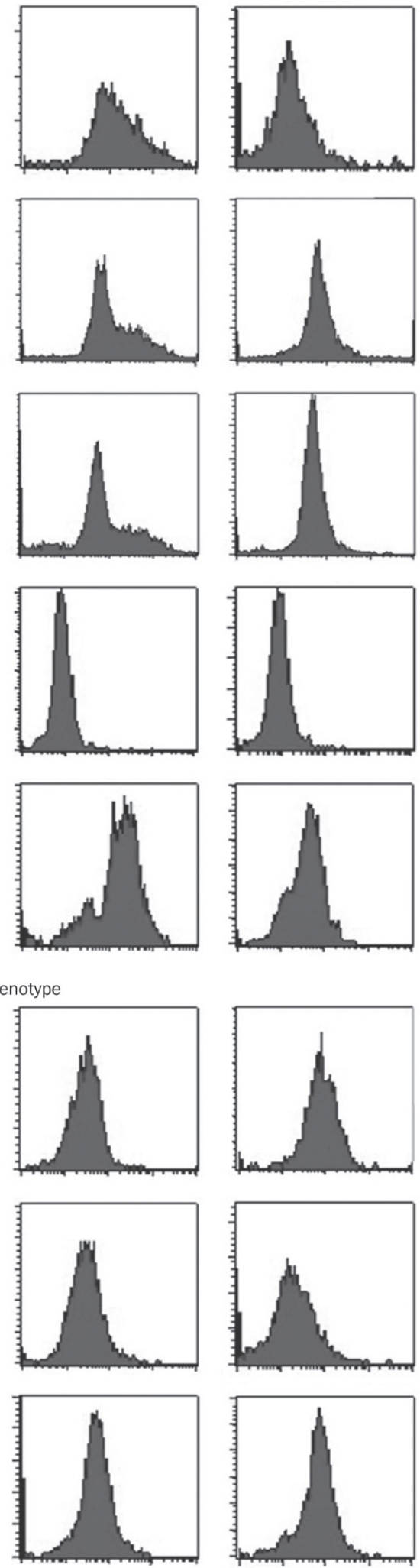

IFN-Y
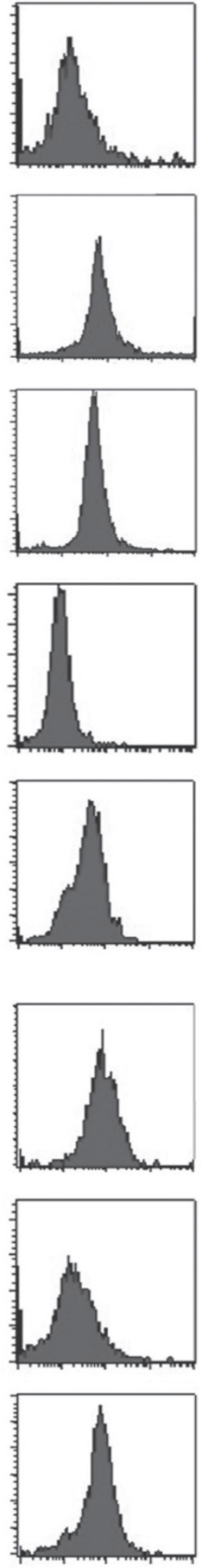

IFN-y+fasudil
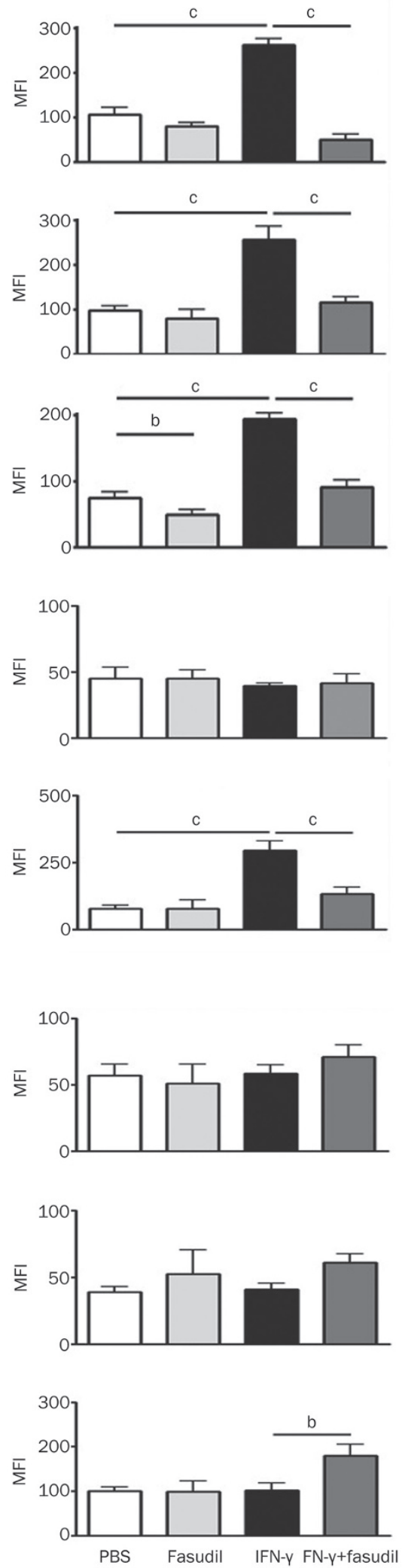

Figure 2. Fasudil shifts M1 macrophages to the M2 phenotype, as studied using flow cytometry. The BV-2 cells were cultured in the absence or presence of IFN-y $(100 \mathrm{U} / \mathrm{mL})$ and/or fasudil $(15 \mu \mathrm{g} / \mathrm{mL})$ for $72 \mathrm{~h}$. The cells were stained with M1 macrophage markers iNOS, MHC class II, CD11c, CCR7, and CD16/32 as well as M2 macrophage markers arginase, CD23 and CD206. After staining and washing, the cells were analyzed at the indicated time points using flow cytometry, according to the CFSE dilution. The results were expressed as the mean fluorescence intensity (MFI) in the histogram. The quantitative analysis was performed based on 3 independent experiments with similar results. ${ }^{b} P<0.05,{ }^{\mathrm{C}} P<0.01$. 
$P<0.05)$.

Next, we attempted to define the effect of fasudil on the polarization of the BV-2 microglia using alternative assays. We measured the expression of M1 iNOS and M2 arginase in the BV-2 microglia using Western blotting. As shown in Figure 3A, the fasudil treatment significantly inhibited IFN- $\gamma-$ stimulated iNOS expression $(P<0.05)$ and slightly induced the expression of arginase. Simultaneously, we measured the levels of nitric oxide (NO) and the activity of arginase in the BV-2 cells by chemical and enzymatic assays. IFN- $\gamma$ stimulated the production of $\mathrm{NO}$ (Figure 3B, $P<0.05$ ), but did not affect the activity of arginase. However, fasudil significantly decreased the production of $\mathrm{NO}$ and enhanced the activity of arginase (Figure $3 \mathrm{~B}, P<0.05$ ). In addition, we detected two representative cytokines, TNF- $\alpha$ for M1 microglia and IL-10 for M2 microglia, using ELISA kits. The results showed that the fasudil treatment significantly inhibited IFN-ү-stimulated TNF-a production (Figure 3B, $P<0.01$ ) and slightly increased IL-10 production (not statistically significant), compared with the IFN-y treatment. Our results clearly indicate that the fasudil treatment can shift the BV-2 microglia from the M1 to the M2 phenotype, but primarily through suppressing the M1 phenotype and function. No difference in the above results was observed after exposure to $15 \mu \mathrm{g} / \mathrm{mL}$ of fasudil, compared with PBS exposure (data not shown).

\section{Fasudil-treated BV-2 microglia induce conversion of encephalo-} myelitic T cells

The next question was whether the polarization of the BV-2 microglia mediated by fasudil could affect the population and function of the encephalomyelitic T cells. Fasudil-treated BV-2 cells were co-cultured with the encephalomyelitic T cells
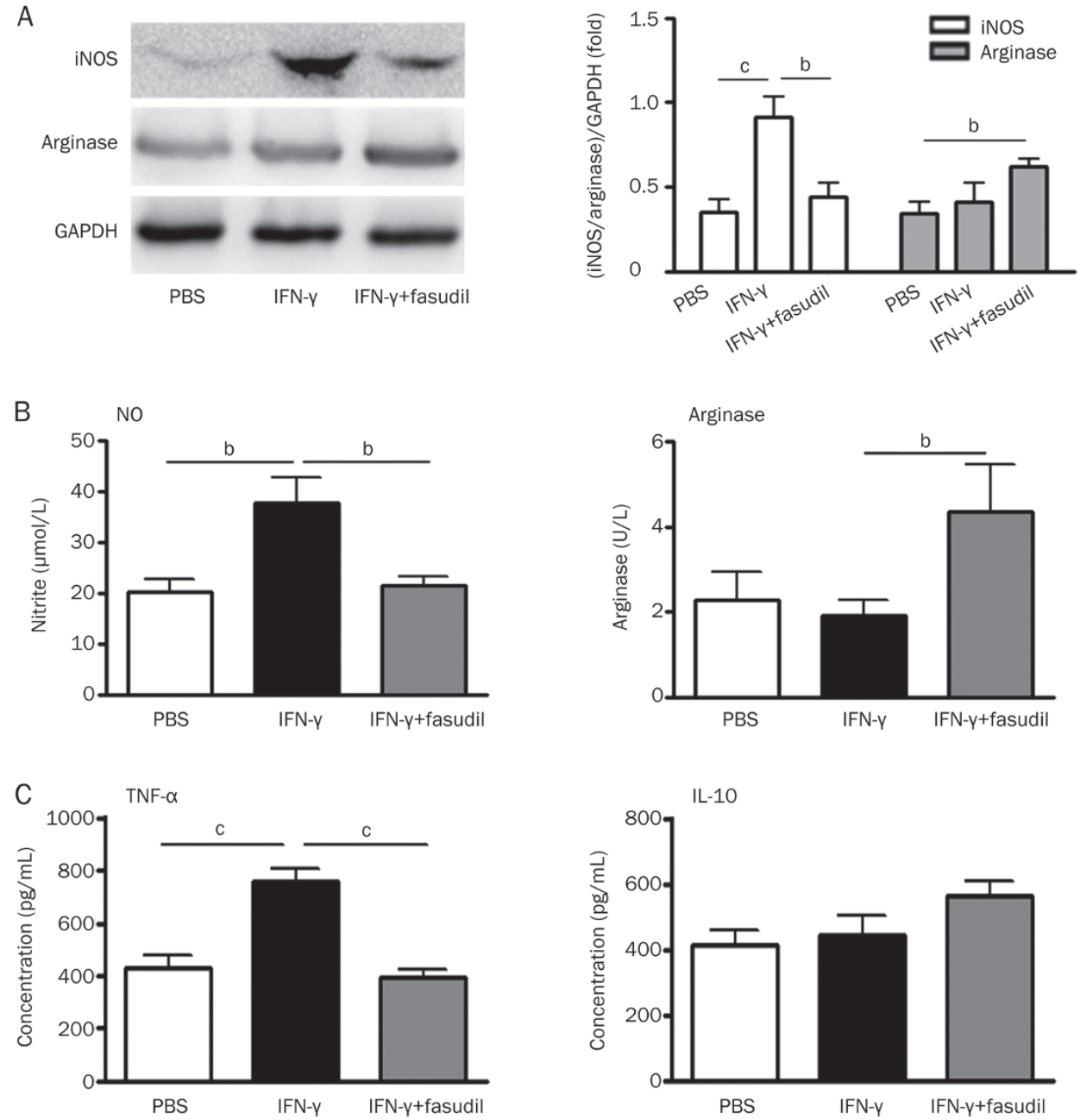

Figure 3. Fasudil shifts $M 1$ to the $M 2$ polarization of BV-2 microglia. The BV-2 cells were cultured in the absence or presence of IFN-y (100 $U / m L$ ) and/or fasudil $(15 \mu \mathrm{g} / \mathrm{mL})$ for $72 \mathrm{~h}$. The protein was extracted from the BV-2 microglia, and the expression of iNOS and arginase was measured using Western blotting (A). The production of NO and the activity of arginase were detected by chemical and enzymatic assays (B). Simultaneously, the levels of TNF- $\alpha$ and IL-10 were detected using ELISA kits (C). The results of the Western blot analysis were expressed as the fold change relative to GAPDH as the loading control, and the levels of cytokines were expressed in $\mathrm{pg} / \mathrm{mL}$. The quantitative analysis was performed based on 3 independent experiments with similar results. ${ }^{\mathrm{b}} P<0.05,{ }^{\mathrm{C}} P<0.01$. 
(BV-2 cells: T cells, 1:5) in the presence of $\mathrm{MOG}_{35-55}(10 \mu \mathrm{g} / \mathrm{mL})$ for $72 \mathrm{~h}$. As shown in Figure 4, the fasudil-treated BV-2 cells induced the conversion of the encephalomyelitic $\mathrm{T}$ cells, reflected by the up-regulation of $\mathrm{CD} 44^{+} \mathrm{CD} 25^{\text {high }} \mathrm{T}$ cells and $\mathrm{CD} 4^{+} \mathrm{IL}-10^{+} \mathrm{T}$ cells, and the down-regulation of $\mathrm{CD} 4^{+} \mathrm{IL}-17^{+} \mathrm{T}$ cells (Figure $4, P<0.05$ for $\mathrm{CD} 4^{+} \mathrm{CD} 25^{\text {high }} \mathrm{T}$ cells and $\mathrm{CD} 4^{+} \mathrm{IL}-10^{+}$ T cells; $P=0.06$ for $\mathrm{CD} 4^{+} \mathrm{IL}-17^{+} \mathrm{T}$ cells).

Next, we determined whether the fasudil-treated BV-2 microglia exert their inhibitory effect on encephalomyelitic $\mathrm{T}$ cell proliferation. The BV-2 cells were co-cultured with encephalomyelitic T cells labeled with CFSE for $72 \mathrm{~h}$ in the presence of $\mathrm{MOG}_{35-55}(10 \mu \mathrm{g} / \mathrm{mL})$. Figure $5 \mathrm{~A}$ showed a typical analysis of the proliferation of the CFSE-labeled T cells. Upon co-culture with the BV-2 cells treated with fasudil, the T cell proliferation was lower than that in the mixed culture of $\mathrm{T}$ cells and BV-2 cells treated with PBS (Figure 5A, $P<0.05$ ). As shown in Figure 5B, fasudil-treated BV-2 microglia inhibited IL-17 production by the T cells and induced them to produce IL-10, compared with the PBS-treated BV-2 microglia (Figure $5 \mathrm{~B}, P<0.05)$. Our results define that the M2 polarization of the BV-2 microglia mediated by fasudil can convert the function of the encephalomyelitic $T$ cells, from encephalomyelitic $T$ cells into Tregs.

\section{Fasudil shifts the phenotype of microglia from M1 to M2 in vivo}

To confirm the effect of fasudil on microglial polarization in vivo, fasudil $\left(40 \mathrm{mg} \cdot \mathrm{kg}^{-1} \cdot \mathrm{d}^{-1}\right)$ or saline was injected ip into the EAE mice induced with $\mathrm{MOG}_{35-55}$. On d $28 \mathrm{pi}$, the expres-
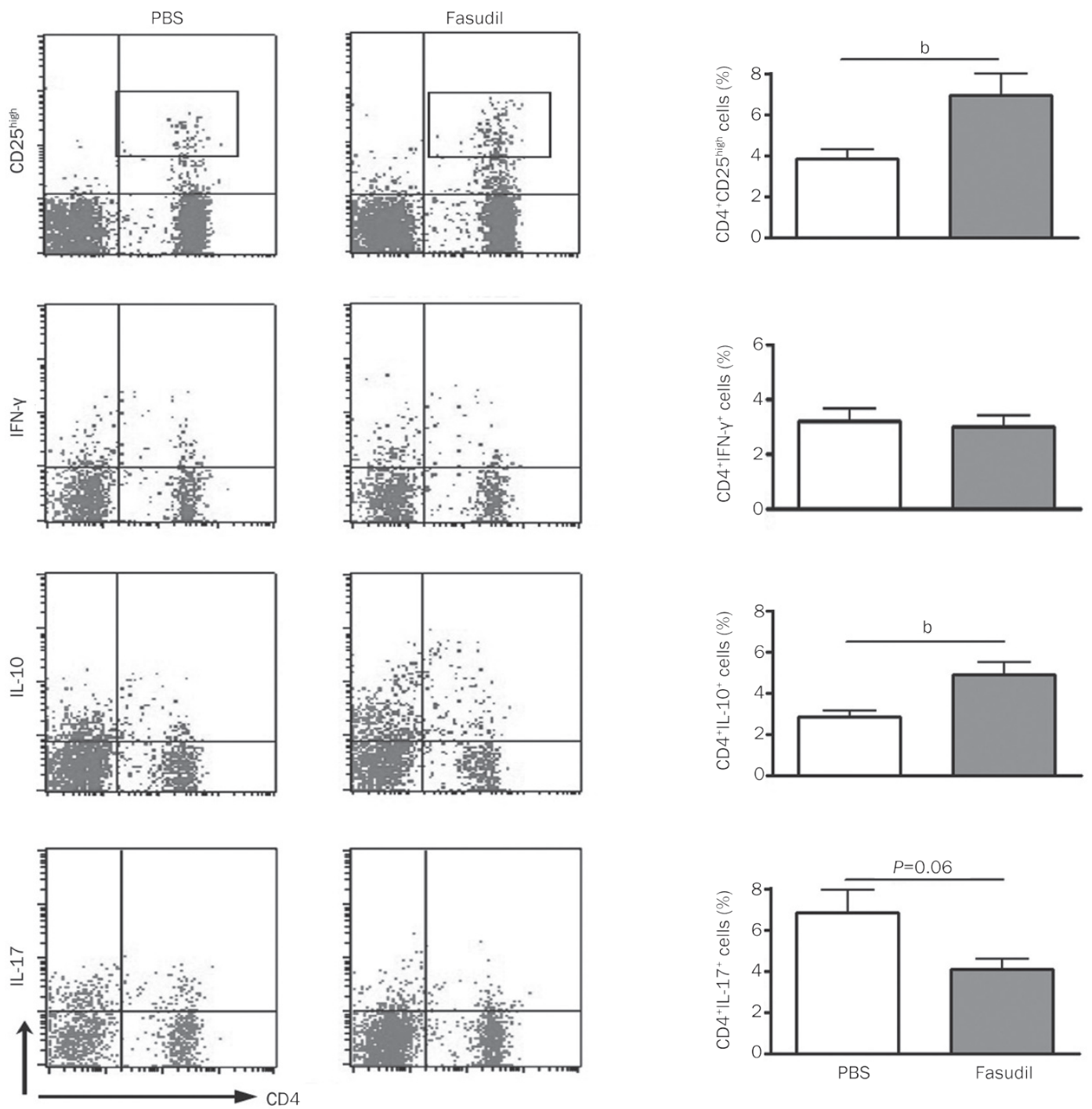

Figure 4. Fasudil-treated BV-2 cells induce the conversion of encephalomyelitic $T$ cells. The encephalitogenic CD4-positive $T$ cells specific for $M_{0} G_{35-55}$ were purified from EAE mice at d 9 post-immunization and co-cultured with PBS- or fasudil-treated BV-2 cells in an estimated ratio of 5:1 in the presence of $\mathrm{MOG}_{35-55}(10 \mu \mathrm{g} / \mathrm{mL})$ for $72 \mathrm{~h}$. The cells were stained with FITC-anti CD4 and PE-anti CD25 or PE-anti IFN-y or PE-anti IL-10 or PE-anti IL-17. After staining and washing, the expression of CD25, IFN-y, IL-10, and IL-17 on CD4 ${ }^{+} \mathrm{T}$ cells were analyzed using flow cytometry. The results were expressed as the percentage of cells in the designated area. The quantitative analysis was performed based on at least 2 independent experiments with similar results. ${ }^{b} P<0.05$. 
A

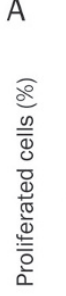

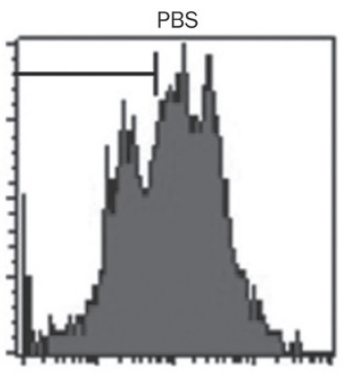

B

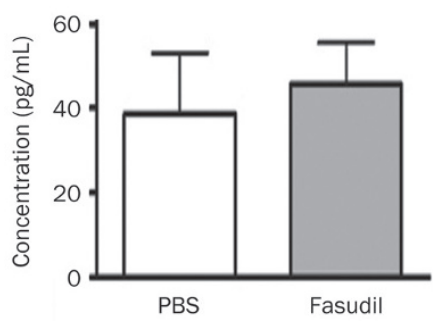

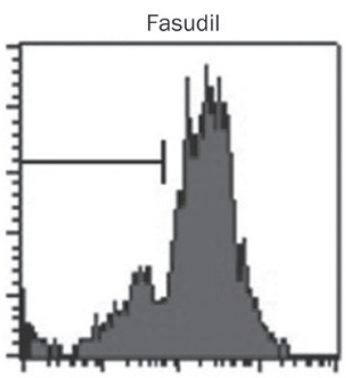

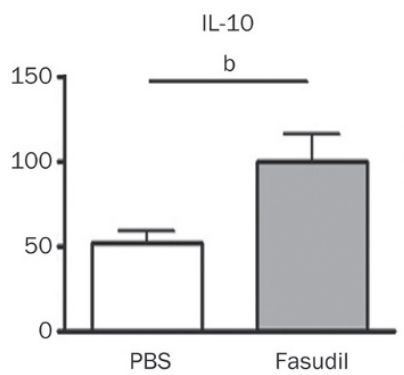

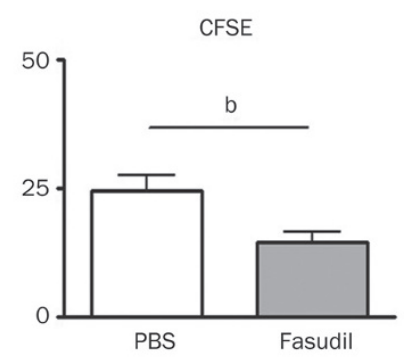

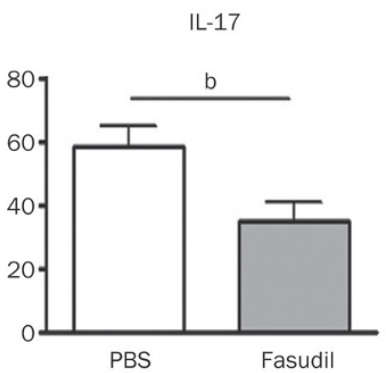

Figure 5. Fasudil-treated BV-2 cells convert inflammatory T cells to regulatory T cells. The encephalitogenic CD4-positive $\mathrm{T}$ cells specific for $\mathrm{MOG}_{35-55}$ were labeled with CFSE and co-cultured with PBS- or fasudil-treated BV-2 cells in an estimated ratio of 5:1 in the presence of $\mathrm{MOG}_{35-55}(10 \mu \mathrm{g} / \mathrm{mL})$ for $72 \mathrm{~h}$. The percentage of proliferating cells was determined through the measurement of CFSE dilution using flow cytometry (A). The levels of IFN-y, IL-10, and IL-17 in the supernatants were detected using ELISA kits. (B) The quantitative analysis was performed based on at least 2 independent experiments with similar results. ${ }^{\mathrm{b}} \mathrm{P}<0.05$.

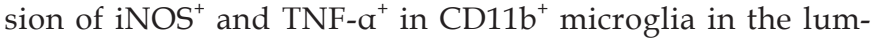
bar enlargement of the spinal cord of fasudil-treated mice was inhibited, compared with that in saline-injected mice, as observed using double immunohistochemistry (Figure 6, $P<0.01$ for iNOS and $P<0.05$ for TNF- $\alpha$ ). By contrast, the mean expression of IL- $10^{+}$in the $\mathrm{CD} 11 \mathrm{~b}^{+}$microglia was enhanced by the fasudil treatment $(P<0.05)$, although the number of $\mathrm{CD}_{11} \mathrm{~b}^{+}$ arginase $^{+}$microglia was not different in saline-treated and fasudil-treated mice (Figure 6). Overall, these results clearly indicate that fasudil could convert M1 microglia to the M2 phenotype both in vitro and in vivo.

\section{Discussion}

MS is a chronic, disabling neurological disease characterized by progressive autoimmune abnormality. Based on studies on the mechanism and pathogenesis, MS treatment involves 3 therapeutic approaches: 1) halting the abnormal immune response that triggers the disease process ${ }^{[28]} ; 2$ ) protecting neurons from further neurodegeneration, as well as axonal damage and deficits ${ }^{[29]}$; and 3) repairing demyelination and restoring neuronal functions by promoting endogenous mechanisms of neuronal repair or by transplanting exogenous myelinating cells $^{[30]}$. However, current FDA-approved treatments focus primarily on immunomodulation and are only partially effective. Thus, alternative therapeutic strategies are necessary, such as neuroprotective and neuroregeneration.

Findings in MS patients and EAE models support the hypothesis that pathogenic $T$ cells migrate and cross the blood brain barrier (BBB), then act in concert with microglia to destroy the central myelin sheaths as well as axons, resulting in the increased degeneration of surrounding neurons ${ }^{[31,32]}$. However, an increasing number of studies indicate that microglia play a dual role ${ }^{[6]}$. Harmful effects include toxicity to neurons and oligodendrocytes, release of proteases/ inflammatory cytokines/free radicals, and re-activation of $\mathrm{T}$ cells in the CNS. Beneficial roles include axon regeneration, remyelination, clearance of myelin debris, and the release of neurotrophic factors ${ }^{[6]}$. Recent research indicates that macrophages/microglia of different phenotypes display completely different biological characteristics ${ }^{[17]}$. In the EAE model, M1 macrophages play a major pathogenetic role in disease initiation, whereas M2 macrophages contribute to disease recovery ${ }^{[33,34]}$. Treatments transforming M1 to M2 macrophages remarkably reduced EAE severity in mice ${ }^{[26]}$, indicating that drugs that induce M2 differentiation are promising therapeutic approaches.

To date, the molecular mechanism of macrophage/microglia polarization is not fully elucidated. Several molecules may be involved in the process of macrophage/microglia polarization. NF-KB and STAT1 activation promotes M1 macrophage polarization, resulting in cytotoxic and inflammatory functions. By contrast, STAT3 and STAT6 activation results in M2 macrophage polarization and is associated with immune suppression. LPS can skew M2 type cells into the M1 type through the p38 MARK pathway ${ }^{[35]}$. Blocking TLR4 signaling increased the anti-inflammatory potential in LPS-stimulated BV-2 microglia ${ }^{[36]}$.

IL-10 activates STAT3, and there is some evidence to suggest 

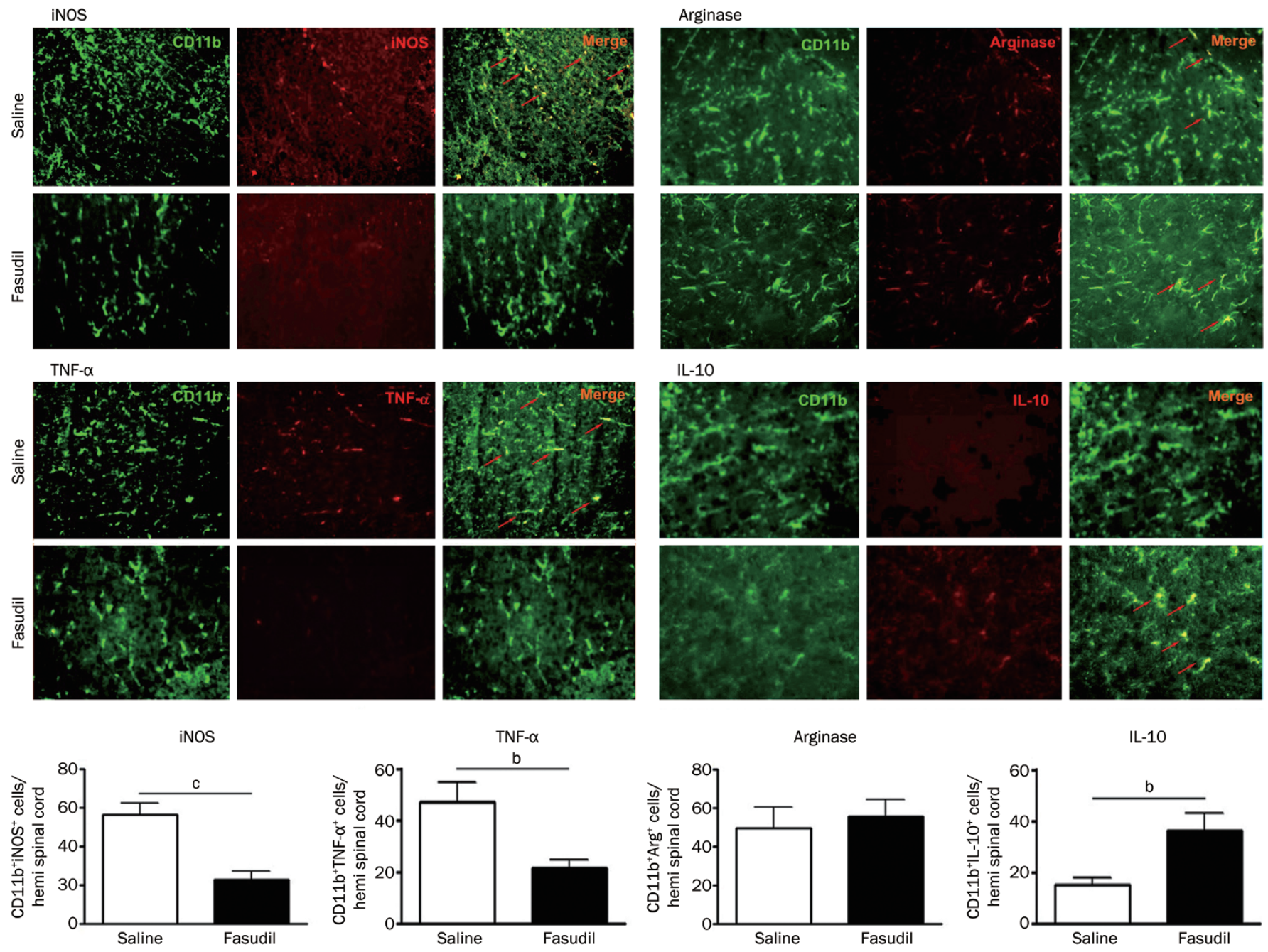

Figure 6. Fasudil shifts M1 to the M2 phenotype in vivo. Chronic EAE was induced in C57BL6 mice with MOG $35-55$ and fasudil was injected (ip) from d 3 pi till d 27 pi. Sections of the spinal cords were co-stained with anti-CD11b and anti-iNOS or anti-TNF- $\alpha$, as well as with anti-CD11b and anti-arginase or anti-IL-10. The number of double-positive cells was observed in the lumbar enlargement of the spinal cord. The representative pattern was obtained from 1 of 3 experiments with similar results. Quantitative analysis was performed for $4-5$ mice in each group. ${ }^{b} P<0.05 ;{ }^{c} P<0.01$.

that STAT3 may also play a role in promoting M2 macrophage polarization $^{[37]}$. In this study, 2 molecules may be involved in shifting the M1 phenotype to M2. First, higher levels of IL-10 derived from $\mathrm{CD}^{+} \mathrm{IL}^{-10^{+}} \mathrm{T}$ cells and M2 BV-2 cells may participate in the conversion of M1 to M2 microglia through an autocrine and/or paracrine manner. Second, the relationship between iNOS and arginase, a competitive enzyme for iNOS, may influence microglia polarization. Macrophage arginase expression is regulated in response to anti-inflammatory cytokines, such as IL-4 and IL-10 ${ }^{[38]}$. Glatiramer acetate promotes an anti-inflammatory M2 phenotype by increased antiinflammatory cytokine IL-10 in macrophages/microglia ${ }^{[39,40]}$. Arginase induced mainly by Th2 cytokines can down-regulate iNOS, exhibiting iNOS-expressing M1 cells into arginaseexpressing M2 cells.

Fasudil treatment stimulated long dendritic formation on BV-2 microglia, compared with PBS or IFN- $\gamma$. Microglia are highly complex cells found in the CNS that exhibit multiple forms and functional specializations, in the form of a variety of cell shapes ranging from an amoeboid to a ramified phenotype. The morphology of microglia is closely related to their functions in the microenvironment: amoeboid, when they eliminate cell debris; ramified, when they keep their microenvironment under surveillance; and branched cytoplasmic extensions, when they make direct contact with synaptic elements to modulate activity. Ramified microglia possess a characteristic form composed of long branching processes that are highly sensitive to alterations in the local microenvironment ${ }^{[41]}$. The function of morphological changes induced with fasudil in BV-2 cells is further investigated, including the formation of neuron synapses and phagocytosis.

In the previous study, we explored the therapeutic effect of fasudil in EAE mice, including reduced inflammatory response and demyelination, possibly through an anti-inflammatory mechanism $^{[24,26]}$. On the one hand, fasudil directly inhibits the infiltration of inflammatory cells into the CNS through 2 
mechanisms: 1) fasudil reduces the permeability of the BBB by inhibiting occludin and claudin- 5 phosphorylation ${ }^{[42]}$ and 2) fasudil inhibits ROCK-mediated T-cell polarization and transendothelial migration by regulating cytoskeletal dynamics and cell adhesion ${ }^{[43]}$. On the other hand, the administration of fasudil may inhibit the CNS inflammatory response by regulating the functional plasticity of microglia, which subsequently affects the balance between proinflammatory and regulatory $\mathrm{T}$ cells and finally contribute to myelin repair and inflammatory suppression. In the present study, we confirmed that fasudil converts M1 microglia, expressing iNOS and TNFa, to M2 microglia, expressing arginase and IL-10, both in vitro and in vivo. In the mixed culture of BV-2 microglia and T cells, fasudil-treated BV-2 microglia promoted the transformation of encephalomyelitic T cells into regulatory/inhibitory $\mathrm{T}$ cells that did not induce the development of EAE (data not shown). Th1 and Th17 cells, the predominant immunological players during EAE/MS development ${ }^{[4,45]}$, are primed by CNSspecific antigens presented by antigen-presenting cells in the peripheral lymphoid organs. Next, these primed T cells cross the $\mathrm{BBB}$ and consequently initiate a local inflammation that impairs myelin sheaths and axons, when encountering their cognate antigen presented by CNS microglia ${ }^{[46,47]}$. In the present study, we provided direct evidence that fasudil converts encephalomyelitic T cells into Tregs through the polarization of M2 BV-2 cells. We speculate that an increased release of IL-10 from M2 microglia and regulatory T cells may convert encephalomyelitic $\mathrm{T}$ cells into Tregs via autocrine and paracrine effects. In addition, other biological factors that affect $\mathrm{T}$ cells are also being explored. Therefore, the M2 polarization of fasudil-stimulated BV-2 microglia may inhibit the CNS inflammatory response through 2 pathways: 1) fasudil directly results in the polarization of inflammatory microglia/ macrophages to anti-inflammatory cells, both in the peripheral immune system and in the CNS microenvironment and 2) fasudil indirectly regulates $\mathrm{T}$ cell responses through antigen presentation of polarized microglia/macrophages.

\section{Conclusion}

Our data demonstrate that fasudil triggers the polarization of inflammatory M1 microglia to anti-inflammatory cells that convert encephalomyelitic $\mathrm{T}$ cells to Tregs. These data partially indicate that fasudil exhibits a therapeutic effect by regulating the polarization of microglia/macrophages and the balance of immune cells during the progression of EAE.

\section{Acknowledgements}

This work was supported by grants from the National Natural Science Foundation of China (№ 81070957, 81371414, and 81070956).

\section{Author contribution}

Chan CHEN, Yan-hua LI, Qiong ZHANG, and Yong-fei ZHAO performed research; Jie-zhong YU and Bao-guo XIAO analyzed data; Chan CHEN, Cun-gen MA, and Bao-guo XIAO wrote the paper.

\section{References}

1 Stangel M. Neurodegeneration and neuroprotection in multiple sclerosis. Curr Pharm Des 2012; 18: 4471-4.

2 Batoulis H, Recks MS, Addicks K, Kuerten S. Experimental autoimmune encephalomyelitis - achievements and prospective advances. APMIS 2011; 119: 819-30.

3 Constantinescu CS, Farooqi N, O'Brien K, Gran B. Experimental autoimmune encephalomyelitis (EAE) as a model for multiple sclerosis (MS). Br J Pharmacol 2011; 164: 1079-106.

4 Fletcher JM, Lalor SJ, Sweeney CM, Tubridy N, Mills KH. T cells in multiple sclerosis and experimental autoimmune encephalomyelitis. Clin Exp Immunol 2010; 162: 1-11.

5 Jadidi-Niaragh F, Mirshafiey A. Th17 cell, the new player of neuroinflammatory process in multiple sclerosis. Scand J Immunol 2011; 74: 1-13.

6 Rawji KS, Yong VW. The benefits and detriments of macrophages/ microglia in models of ultiple sclerosis. Clin Dev Immunol 2013; 2013: 948976.

7 Dutra RC, Bento AF, Leite DF, Manjavachi MN, Marcon R, Bicca MA, et al. The role of kinin B1 and B2 receptors in the persistent pain induced by experimental autoimmune encephalomyelitis (EAE) in mice: evidence for the involvement of astrocytes. Neurobiol Dis 2013; 54: 82-93.

8 Chastain EM, Duncan DS, Rodgers JM, Miller SD. The role of antigen presenting cells in multiple sclerosis. Biochim Biophys Acta 2011; 1812: 265-74.

9 Benveniste EN. Role of macrophages/microglia in multiple sclerosis and experimental allergic encephalomyelitis. J Mol Med 1997; 75: 165-73.

10 Sloka S. Reduction of microglial activity in a model of multiple sclerosis by dipyridamole. J Neuroinflammation 2013; 10: 89.

11 Prineas JW, Parratt JD. Oligodendrocytes and the early multiple sclerosis lesion. Ann Neurol 2012; 72: 18-31.

12 Pang Y, Campbell L, Zheng B, Fan L, Cai Z, Rhodes P. LPS-activated microglia induce death of oligodendrocyte progenitor cells and impede their development. Neuroscience 2010; 166: 464-75.

13 Heppner FL, Greter M, Marino D, Falsig J, Raivich G, Hövelmeyer N, et al. Experimental autoimmune encephalomyelitis repressed by microglial paralysis. Nat Med 2005; 11: 146-52.

14 Napoli I, Neumann H. Protective effects of microglia in multiple sclerosis. Exp Neurol 2010; 225: 24-8.

15 Girard S, Brough D, Lopez-Castejon G, Giles J, Rothwell NJ, Allan SM. Microglia and macrophages differentially modulate cell death after brain injury caused by oxygen-glucose deprivation in organotypic brain slices. Glia 2013; 61: 813-24.

16 Ajmone-Cat MA, Mancini M, De Simone R, Cilli P, Minghetti L. Microglial polarization and plasticity: evidence from organotypic hippocampal slice cultures. Glia 2013; 61: 1698-711.

17 Cao L, He C. Polarization of macrophages and microglia in inflammatory demyelination. Neurosci Bull 2013; 29: 189-98.

18 Miron VE, Boyd A, Zhao JW, Yuen TJ, Ruckh JM, Shadrach JL, et al. M2 microglia and macrophages drive oligodendrocyte differentiation during CNS remyelination. Nat Neurosci 2013; 16: 1211-8.

19 Martinez-Pasamar S, Abad E, Moreno B, Velez de Mendizabal N, Martinez-Forero I, Garcia-Ojalvo J, et al. Dynamic cross-regulation of antigen-specific effector and regulatory $\mathrm{T}$ cell subpopulations and $\mathrm{mi}-$ croglia in brain autoimmunity. BMC Syst Biol 2013; 7: 34.

20 Jaini R, Popescu DC, Flask CA, Macklin WB, Tuohy VK. Myelin antigen load influences antigen presentation and severity of central nervous system autoimmunity. J Neuroimmunol 2013; 259: 37-46.

21 Suzuki Y, Shibuya M, Satoh S, Sugiyama H, Seto M, Takakura K. 
Safety and efficacy of fasudil monotherapy and fasudil-ozagrel combination therapy in patients with subarachnoid hemorrhage: subanalysis of the post-marketing surveillance study. Neurol Med Chir (Tokyo) 2008; 48: 241-7.

22 Shibuya M, Hirai S, Seto M, Satoh S, Ohtomo E. Fasudil Ischemic Stroke Study Group. Effects of fasudil in acute ischemic stroke: results of a prospective placebo-controlled double-blind trial. J Neurol Sci 2005; 238: 31-9.

23 Hara M, Takayasu M, Watanabe K, Noda A, Takagi T, Suzuki Y, et al. Protein kinase inhibition by fasudil hydrochloride promotes neurological recovery after spinal cord injury in rats. J Neurosurg 2000; 93 : 94-101.

24 Hou SW, Liu CY, Li YH, Yu JZ, Feng L, Liu YT, et al. Fasudil ameliorates disease progression in experimental autoimmune encephalomyelitis, acting possibly through antiinflammatory effect. CNS Neurosci Ther 2012; 18: 909-17.

25 Sun X, Minohara M, Kikuchi H, Ishizu T, Tanaka M, Piao H, et al. The selective Rho-kinase inhibitor Fasudil is protective and therapeutic in experimental autoimmune encephalomyelitis. J Neuroimmunol 2006; 180: 126-34.

26 Liu C, Li Y, Yu J, Feng L, Hou S, Liu Y, et al. Targeting the shift from M1 to M2 macrophages in experimental autoimmune encephalomyelitis mice treated with fasudil. PLoS One 2013; 8: e54841.

27 Bocchini V, Mazzolla R, Barluzzi R, Blasi E, Sick P, Kettenmann H. An immortalized cell line expresses properties of activated micro glial cells. J Neurosci Res 1992; 31: 616-21.

28 Owens T, Tran E, Hassan-Zahraee M, Krakowski M. Immune cell entry to the CNS - a focus for immunoregulation of EAE. Res Immunol 1998; 149: 781-9.

29 Karussis D, Grigoriadis S, Polyzoidou E, Grigoriadis N, Slavin S, Abramsky O. Neuroprotection in multiple sclerosis. Clin Neurol Neurosurg 2006; 108: 250-4.

30 IKassis I, Petrou P, Halimi M, Karussis D. Mesenchymal stem cells (MSC) derived from mice with experimental autoimmune encephalomyelitis (EAE) suppress EAE and have similar biological properties with MSC from healthy donors. Immunol Lett 2013; 154: 70-6.

31 Imitola J, Côté D, Rasmussen S, Xie XS, Liu Y, Chitnis T, et al. Multimodal coherent anti-Stokes Raman scattering microscopy reveals microglia-associated myelin and axonal dysfunction in multiple sclerosis-like lesions in mice. J Biomed Opt 2011; 16: 021109.

32 Yeo YA, Martínez Gómez JM, Croxford JL, Gasser S, Ling EA, Schwarz H. CD137 ligand activated microglia induces oligodendrocyte apoptosis via reactive oxygen species. J Neuroinflammation 2012; 9: 173.

33 Mikita J, Dubourdieu-Cassagno N, Deloire MS, Vekris A, Biran M, Raffard G, et al. Altered M1/M2 activation patterns of monocytes in severe relapsing experimental rat model of multiple sclerosis: amelioration of clinical status by M2 activated monocyte administration. Mult Scler 2011; 17: 2-15.

34 Jiang HR, Milovanović M, Allan D, Niedbala W, Besnard AG, Fukada SY, et al. IL-33 attenuates EAE by suppressing IL-17 and IFN-y production and inducing alternatively activated macrophages. Eur J Immunol 2012; 42: 1804-14.

35 Yang Y, Zhang R, Xia F, Zou T, Huang A, Xiong S, et al. LPS converts Gr- $1^{+} \mathrm{CD} 115^{+}$myeloid-derived suppressor cells from M2 to M1 via P38 MAPK. Exp Cell Res 2013; 319: 1774-83.

36 Jeong JW, Lee HH, Han MH, Kim GY, Kim WJ, Choi YH. Antiinflammatory effects of genistein via suppression of the toll-like receptor 4-mediated signaling pathway in lipopolysaccharidestimulated BV2 microglia. Chem Biol Interact 2014; 212: 30-9.

37 Sica A, Mantovani A. Macrophage plasticity and polarization: in vivo veritas. J Clin Invest 2012; 122: 787-95.

38 Pauleau AL, Rutschman R, Lang R, Pernis A, Watowich SS, Murray PJ. Enhancer-mediated control of macrophage-specific arginase-1 expression. J Immunol 2004; 172: 7565-73.

39 Jung S, Siglienti I, Grauer O, Magnus T, Scarlato G, Toyka K. Induction of IL-10 in rat peritoneal macrophages and dendritic cells by glatiramer acetate. J Neuroimmunol 2004; 148: 63-73.

40 Weber MS, Starck M, Wagenpfeil S, Meinl E, Hohlfeld R, Farina C. Multiple sclerosis: Glatiramer acetate inhibits monocyte reactivity in vitro and in vivo. Brain 2004; 127: 1370-8.

41 Fetler L, Amigorena S. Neurosclence. Brain under surveillance: the microglia patrol. Science 2005; 309: 392-3.

42 Yamamoto M, Ramirez SH, Sato S, Kiyota T, Cerny RI. Phosphorylation of claudin-5 and occludin by rho kinase in brain endothelial cells. Am J Pathol 2008; 172: 521-33.

43 Heasman SJ, Ridley AJ. Multiple roles for RhoA during T cell transendothelial migration. Small GTPases 2010; 1: 174-9.

44 El-behi M, Rostami A, Ciric B. Current views on the roles of Th1 and Th17 cells in experimental autoimmune encephalomyelitis. J Neuroimmune Pharmacol 2010; 5: 189-97.

45 Pierson E, Simmons SB, Castelli L, Goverman JM. Mechanisms regulating regional localization of inflammation during CNS autoimmunity. Immunol Rev 2012; 248: 205-15.

46 Almolda B, Gonzalez B, Castellano B. Antigen presentation in EAE: role of microglia, macrophages and dendritic cells. Front Biosci 2011; 16: $1157-71$.

47 Kawakami N, Bartholomäus I, Pesic M, Mues M. An autoimmunity odyssey: how autoreactive T cells infiltrate into the CNS. Immunol Rev 2012; 248: 140-55. 